\title{
Chiral phase transition in a covariant nonlocal NJL model
}

\author{
I. General ${ }^{\mathrm{a}}$, D. Gomez Dumm ${ }^{\mathrm{b}, 1}$, N.N. Scoccola ${ }^{\mathrm{a}, \mathrm{c}, 1}$ \\ ${ }^{a}$ Physics Department, Comisión Nacional de Energía Atómica, Av. Libertador 8250, (1429) Buenos Aires, Argentina \\ ${ }^{\mathrm{b}}$ IFLP, Departamento de Física, Universidad Nacional de La Plata, C.C. 67, (1900) La Plata, Argentina \\ c Universidad Favaloro, Solís 453, (1078) Buenos Aires, Argentina
}

Received 23 October 2000; received in revised form 8 January 2001; accepted 31 January 2001

Editor: J.-P. Blaizot

\begin{abstract}
The properties of the chiral phase transition at finite temperature and chemical potential are investigated within a nonlocal covariant extension of the Nambu-Jona-Lasinio model based on a separable quark-quark interaction. We consider both the situation in which the Minkowski quark propagator has poles at real energies and the case where only complex poles appear. In the literature, the latter has been proposed as a realization of confinement. In both cases, the behaviour of the physical quantities as functions of $T$ and $\mu$ is found to be quite similar. In particular, for low values of $T$ the chiral transition is always of first order and, for finite quark masses, at certain "end point" the transition turns into a smooth crossover. In the chiral limit, this "end point" becomes a "tricritical" point. Our predictions for the position of these points are similar, although somewhat smaller, than previous estimates. Finally, the relation between the deconfining transition and chiral restoration is also discussed. ๑ 2001 Published by Elsevier Science B.V.
\end{abstract}

PACS: $12.39 . \mathrm{Ki} ; 11.30 . \mathrm{Rd} ; 11.10 . \mathrm{Wx}$

Keywords: Nonlocal Nambu-Jona-Lasinio model; Finite temperature and/or density; Chiral phase transition

The behaviour of hot dense hadronic matter and its transition to a plasma of quarks and gluons has received considerable attention in recent years. To a great extent this is motivated by the advent of facilities like, e.g., RHIC at Brookhaven which are expected to provide some empirical information about such transition. The interest in this topic has been further increased by the recent suggestions that the QCD phase diagram could be richer than previously expected (see Ref. [1] for some recent review articles). Due to the well known difficulties to deal directly with QCD, different models have been used to study this sort of problems. Among them the Nambu-JonaLasinio model [2] is one of the most popular. In this model the quark fields interact via local four point vertices which are subject to chiral symmetry. If such interaction is strong enough the chiral symmetry is spontaneously broken and pseudoscalar Goldstone bosons appear. It has been shown by many authors that when the temperature and/or density increase, the chiral symmetry is restored [3]. For zero chemical potential and finite temperature this transition is found to be a smooth one. However, whether for finite chemical potential and zero temperature the transition is of first order or not is highly dependent on the parameters of the model and on the approximations

E-mail address: dumm@venus.fisica.unlp.edu.ar (D. Gomez Dumm).

1 Fellow of CONICET, Argentina. 
made. For example, in the $S U(2)$ version of the NJL model a Hartree-Fock treatment leads to a first order transition, while the Hartree treatment as well as the $S U(3)$ model calculations seem to favor a second order one [3]. On the other hand, several recent calculations performed within various other models [4-6] clearly suggest that in QCD this transition should be of first order. Interestingly, the model used in Ref. [5] can be understood as a nonlocal generalization of the NJL. However, the corresponding four point vertex is local in time and, therefore, not covariant. Some covariant nonlocal extensions of the NJL model have been studied in the last few years [7]. Nonlocality arises naturally in the context of several of the most successful approaches to low-energy quark dynamics as, for example, the instanton liquid model [8] and the Schwinger-Dyson resummation techniques [9]. It has been also argued that nonlocal covariant extensions of the NJL model have several advantages over the local scheme. Namely, nonlocal interactions regularize the model in such a way that anomalies are preserved [10] and charges properly quantized, the effective interaction is finite to all orders in the loop expansion and, therefore, there is not need to introduce extra cut-offs, soft regulators such as Gaussian functions lead to small NLO corrections [11], etc. In addition, it has been shown [12] that a proper choice of the nonlocal regulator and the model parameters can lead to some form of quark confinement, in the sense of a quark propagator without poles at real energies. Recently, the behaviour of this kind of models at finite temperature has been investigated [13]. In this Letter we extend such studies to finite temperature and chemical potential.

We consider a nonlocal extension of the $S U(2)$ NJL model defined by the effective action

$$
\begin{aligned}
S= & \int d^{4} x \bar{\psi}(x)\left(i \not \partial-m_{c}\right) \psi(x)+\int d^{4} x_{1} \cdots d^{4} x_{4} V\left(x_{1}, x_{2}, x_{3}, x_{4}\right) \\
& \times\left(\bar{\psi}\left(x_{1}\right) \psi\left(x_{3}\right) \bar{\psi}\left(x_{2}\right) \psi\left(x_{4}\right)+\bar{\psi}\left(x_{1}\right) i \gamma_{5} \tau^{a} \psi\left(x_{3}\right) \bar{\psi}\left(x_{2}\right) i \gamma_{5} \tau^{a} \psi\left(x_{4}\right)\right),
\end{aligned}
$$

where $m_{c}$ is the (small) current quark mass responsible for the explicit chiral symmetry breaking. The interaction kernel in Euclidean momentum space is given by

$$
V\left(q_{1}, q_{2}, q_{3}, q_{4}\right)=\frac{(2 \pi)^{4}}{2} G r\left(q_{1}^{2}\right) r\left(q_{2}^{2}\right) r\left(q_{3}^{2}\right) r\left(q_{4}^{2}\right) \delta\left(q_{1}+q_{2}-q_{3}-q_{4}\right),
$$

where $r\left(q^{2}\right)$ is a regulator normalized in such a way that $r(0)=1$. Some general forms for this regulator like Lorentzian or Gaussian functions have been used in the literature. A particular form is given, e.g., in the case of instanton liquid models.

Like in the local version of the NJL model, the chiral symmetry is spontaneously broken in this nonlocal scheme for large enough values of the coupling $G$. In the Hartree approximation the self-energy $\Sigma\left(q^{2}\right)$ at vanishing temperature and chemical potential is given by

$$
\Sigma\left(q^{2}\right)=m_{c}+\left(\Sigma(0)-m_{c}\right) r^{2}\left(q^{2}\right),
$$

where the zero-momentum self-energy $\Sigma(0)$ is a solution of the gap equation

$$
\frac{2 \pi^{4}}{G N_{c}}\left(\Sigma(0)-m_{c}\right)=\int d^{4} q \frac{\left[m_{c}+\left(\Sigma(0)-m_{c}\right) r^{2}\left(q^{2}\right)\right] r^{2}\left(q^{2}\right)}{q^{2}+\left[m_{c}+\left(\Sigma(0)-m_{c}\right) r^{2}\left(q^{2}\right)\right]^{2}} .
$$

In general, the quark propagator might have a rather complicate structure of poles and cuts in the complex plane. In what follows we will assume that the regulator is such that it only has an arbitrary but numerable set of poles. As already mentioned, the absence of purely imaginary poles in the Euclidean quark propagator might be interpreted as a realization of confinement [12]. In that case quartets of poles located at $\alpha_{p}=R_{p} \pm i I_{p}, \alpha_{p}=-R_{p} \pm i I_{p}$ appear. On the other hand, if purely imaginary poles exist they will show up as doublets $\alpha_{p}= \pm i I_{p}$. It is clear that the number and position of the poles depend on the details of the regulator. For example, if we assume it to be a step function as in the standard NJL model only two purely imaginary poles at $\pm i M$ appear, with $M$ being the dynamical quark mass. For a Gaussian interaction, three different situations might occur. For values of $\Sigma(0)$ below a certain critical value $\Sigma(0)_{\text {crit }}$ two pairs of purely imaginary simple poles and an infinite set of quartets 
of complex simple poles appear. At $\Sigma(0)=\Sigma(0)_{\text {crit }}$, the two pairs of purely imaginary simple poles turn into a doublet of double poles with $R_{p}=0$, while for $\Sigma(0)>\Sigma(0)_{\text {crit }}$ only an infinite set of quartets of complex simple poles is obtained. For the Lorentzian interactions there is also a critical value above which purely imaginary poles cease to exist. However, for this family of regulators the total number of poles is always finite.

To introduce finite temperature and chemical potential we follow the imaginary time formalism. Thus, we replace the fourth component of the Euclidean quark momentum by $\omega_{n}-i \mu$, where $\omega_{n}=(2 n+1) \pi T$ are the discrete Matsubara frequencies and $\mu$ is the chemical potential. In what follows we will assume that the temperature and chemical potential dependences enter only through those quantities that carry a $q^{2}$-dependence at $T=\mu=0$. That is to say, we will consider that the model parameters $G$ and $m_{c}$, as well as the shape of the regulator, do not change with $T$ or $\mu$. Performing this replacement in the gap equation Eq. (4) we get

$$
\frac{2 \pi^{4}}{G N_{c}}\left(\Sigma(0)-m_{c}\right)=2 \pi T \int d^{3} \vec{q} \sum_{n=-\infty}^{n=\infty} \mathcal{F}\left(q_{i \omega_{n}}\right),
$$

where

$$
\mathcal{F}(q)=\frac{\Sigma\left(q^{2}\right) r^{2}\left(q^{2}\right)}{q^{2}+\Sigma^{2}\left(q^{2}\right)}
$$

and $q_{z}^{2}=(-i z-i \mu)^{2}+\vec{q}^{2}$. The sum over $n$ can be expressed in terms of a sum over the poles of $\mathcal{F}\left(q_{z}\right)$, that we denote $z_{p}$, by introducing the auxiliary function $f(z)=1 /(1+\exp (z / T))$ and using the standard techniques described, e.g., in Ref. [14]. It is easy to see that the poles of $\mathcal{F}\left(q_{z}\right)$ are closely related to those of the quark propagator $\alpha_{p}$. For the pair $\alpha_{p}= \pm\left(R_{p}+i I_{p}\right)$ the associated values are $z_{p}= \pm \epsilon_{p}-\mu \mp i R_{p} I_{p} / \epsilon_{p}$, while for $\alpha_{p}= \pm\left(R_{p}-i I_{p}\right)$ one has $z_{p}= \pm \epsilon_{p}-\mu \pm i R_{p} I_{p} / \epsilon_{p}$, where $\epsilon_{p}$ is defined as

$$
\epsilon_{p}=\sqrt{\frac{I_{p}^{2}-R_{p}^{2}+\vec{q}^{2}+\sqrt{\left(I_{p}^{2}-R_{p}^{2}+\vec{q}^{2}\right)^{2}+4 R_{p}^{2} I_{p}^{2}}}{2}} .
$$

Assuming that all the poles are simple ${ }^{2}$ and performing explicitly the sum over all the poles in a given multiplet we get

$$
\begin{aligned}
& T \int d^{3} \vec{q} \sum_{n=-\infty}^{n=\infty} \mathcal{F}\left(q_{i \omega_{n}}\right) \\
& \quad=\frac{1}{2 \pi} \int d^{4} q \mathcal{F}(q)-\int d^{3} \vec{q} \sum_{\alpha_{p}}^{\prime} \gamma_{p} \operatorname{Re}\left[\operatorname{Res}\left[2 z \mathcal{F}(z) ; \alpha_{p}\right] \frac{\epsilon_{p}}{\epsilon_{p}^{2}+i R_{p} I_{p}}\left(n_{+}+n_{-}\right)\right],
\end{aligned}
$$

where $\operatorname{Res}\left[2 z \mathcal{F}(z) ; \alpha_{p}\right]$ stands for the residue of the function $2 z \mathcal{F}(z)$ evaluated at $z=\alpha_{p}$, and the prime in the sum indicates that it runs over all the poles $\alpha_{p}=R_{p}+i I_{p}$ with $R_{p} \geqslant 0$ and $I_{p}>0$. The coefficient $\gamma_{p}$ is defined as $\gamma_{p}=1 / 2$ for $R_{p}=0$ and $\gamma_{p}=1$ otherwise. The generalized occupation numbers $n_{ \pm}$are given by

$$
n_{ \pm}=\left[1+\exp \left(\frac{\epsilon_{p} \mp \mu+i R_{p} I_{p} / \epsilon_{p}}{T}\right)\right]^{-1} .
$$

As customary, in writing Eq. (8) we have isolated a term which has the same form as the $T=\mu=0$ expression. In this way, all the $T$ and $\mu$ dependent contributions remain finite. Replacing Eq. (8) in the right hand side of Eq. (5)

\footnotetext{
2 This assumption is made just to keep our expressions into a simple form. The generalization to poles of arbitrary multiplicity is rather straightforward.
} 
and using

$$
\operatorname{Res}\left[2 z \mathcal{F}(z) ; \alpha_{p}\right]=\left.\frac{\Sigma(u) r^{2}(u)}{1+\partial_{u} \Sigma^{2}(u)}\right|_{u=\alpha_{p}^{2}},
$$

we obtain the final form of the gap equation at finite temperature and chemical potential. As we see the dependence on these quantities is completely fixed by the pole structure of the quark propagator.

The other quantities which are of interest to understand the characteristics of the chiral phase transition are the quark condensate $\langle\bar{q} q\rangle$ and the quark density $\left\langle q^{\dagger} q\right\rangle$ for each flavour. At $T=\mu=0$, the condensate is given by

$$
\langle\bar{q} q\rangle=-\int \frac{d^{4} q}{(2 \pi)^{4}} \operatorname{Tr}\left[\left(\not q+\Sigma\left(q^{2}\right)\right)^{-1}\right] .
$$

Following similar steps as before, the corresponding result for finite $T$ and $\mu$ can be cast into the form

$$
\begin{aligned}
\langle\bar{q} q\rangle= & -\frac{N_{c}}{4 \pi^{4}} \int d^{4} q \frac{\Sigma\left(q^{2}\right)}{q^{2}+\Sigma^{2}\left(q^{2}\right)} \\
& +\frac{N_{c}}{2 \pi^{3}} \int d^{3} \vec{q} \sum_{\alpha_{p}}^{\prime} \gamma_{p} \operatorname{Re}\left[\left.\frac{\Sigma(u)}{1+\partial_{u} \Sigma^{2}(u)}\right|_{u=\alpha_{p}^{2}} \frac{\epsilon_{p}}{\epsilon_{p}^{2}+i R_{p} I_{p}}\left(n_{+}+n_{-}\right)\right] .
\end{aligned}
$$

Away from the chiral limit this expression turns out to be divergent. Following the standard procedure, we regularize it by subtracting the value obtained in the absence of interactions.

To determine the quark density one has to be more careful due to the presence of nonlocal interactions. In fact, they imply the existence of extra contributions to the Noether currents. The proper expression for the quark density in the Hartree approximation reads

$$
\left\langle q^{\dagger} q\right\rangle=-i \int \frac{d^{4} q}{(2 \pi)^{4}} \operatorname{Tr}\left[\left(q+\Sigma\left(q^{2}\right)\right)^{-1}\left(\gamma_{4}+\partial_{q_{4}} \Sigma\left(q^{2}\right)\right)\right] .
$$

It is seen here that, for any quark flavour, the residue of the pole given by the dressed quark propagator is equal to one. As shown in Ref. [15], this leads to the correct normalization of the baryon number, independently of the shape of the regulator. As above, the result in Eq. (13) can be now extended to finite temperature and chemical potential by replacing $q_{4} \rightarrow \omega_{n}-i \mu$ and summing over all Matsubara frequencies $\omega_{n}$. In this case we obtain

$$
\left\langle q^{\dagger} q\right\rangle=\frac{N_{c}}{2 \pi^{3}} \int d^{3} \vec{q} \sum_{\alpha_{p}}^{\prime} \gamma_{p} \operatorname{Re}\left[n_{+}-n_{-}\right]
$$

Having introduced the formalism needed to extend the model to finite temperature and chemical potential we turn now to our numerical calculations. In this work we take the nonlocal regulator to be of the Gaussian form

$$
r\left(q^{2}\right)=\exp \left(-\frac{q^{2}}{2 \Lambda^{2}}\right)
$$

and consider two sets of values for the parameters of the model. Set I corresponds to $G=50 \mathrm{GeV}^{-2}, m_{c}=$ $10.5 \mathrm{MeV}$ and $\Lambda=627 \mathrm{MeV}$, while for Set II the respective values are $G=30 \mathrm{GeV}^{-2}, m_{c}=7.7 \mathrm{MeV}$ and $\Lambda=760 \mathrm{MeV}$. Both sets of parameters lead to the physical values of the pion mass and decay constant. For Set I the calculated value of the chiral quark condensate at zero temperature and chemical potential is $-(200 \mathrm{MeV})^{3}$ while for Set II it is $-(220 \mathrm{MeV})^{3}$. These values are similar in size to those determined from lattice gauge theory or QCD sum rules. The corresponding results for the self-energy at zero momentum are $\Sigma(0)=350 \mathrm{MeV}$ for Set I and $\Sigma(0)=300 \mathrm{MeV}$ for Set II. Using the explicit expression of $\Sigma(0)_{\text {crit }}$ for the Gaussian interaction,

$$
\Sigma(0)_{\text {crit }}=m_{c}+\frac{1}{2}\left(\sqrt{m_{c}^{2}+2 \Lambda^{2}}-m_{c}\right) \exp \left[-\frac{\left(\sqrt{m_{c}^{2}+2 \Lambda^{2}}+m_{c}\right)^{2}}{4 \Lambda^{2}}\right],
$$


it is easy to check that Set I corresponds to a situation in which there are no purely imaginary poles of the Euclidean quark propagator and Set II to the case in which there are two pairs of them. Therefore, we will also refer to Set I as the confining set and to Set II as the non-confining one.

The behaviour of the zero-momentum self-energy $\Sigma(0)$, the chiral quark condensate $\langle\bar{q} q\rangle$ and the quark density $\rho=\left\langle q^{\dagger} q\right\rangle$ as functions of the chemical potential for some values of the temperature is shown in Fig. 1 . The left and right panels in the figure correspond to the results for Set I and Set II, respectively. In both cases we observe the existence of some kind of phase transition at (or around) a given value of the chemical potential which depends on the temperature. To obtain our results, we have included in the sums over $\alpha_{p}$ appearing in Eqs. (8), (12) and (14) the first few poles of the quark propagator. We have checked, however, that for the range of values of $T$ and $\mu$ covered in our calculations, the convergence is so fast that already the first pole gives almost $100 \%$ of the full result. Thus, the behaviour of relevant physical quantities up to (and somewhat above) the phase transition is basically dominated by the first pole of the quark propagator.

We observe in Fig. 1 that at $T=0$ there is a first order phase transition for both the confining and the nonconfining sets of parameters. As the temperature increases, the value of the chemical potential at which the transition shows up decreases. Finally, above a certain value of the temperature the first order phase transition does not longer exist and, instead, there is a smooth crossover. This phenomenon is clearly shown in the right panel of Fig. 2, where we display the critical temperature at which the phase transition occurs as a function of the chemical potential. The point at which the first order phase transition ceases to exist is usually called "end point". In the chiral limit the latter turns into the so-called "tricritical point", which is the point at which the second order phase transition expected to happen in QCD with two massless quarks becomes a first order one. In fact, this is also what happens within the present model in the chiral limit, as it is shown in the left panel of Fig. 2. Some predictions $[5,6]$ about both the position of this point and its possible experimental signatures [16] exist in the literature. In our case the "tricritical point" is located at $\left(T_{P}, \mu_{P}\right)=(70 \mathrm{MeV}, 130 \mathrm{MeV})$ for Set I and $(70 \mathrm{MeV}, 140 \mathrm{MeV})$ for Set II, while the "end points" are placed at $\left(T_{E}, \mu_{E}\right)=(70 \mathrm{MeV}, 180 \mathrm{MeV})$ and $(55 \mathrm{MeV}, 210 \mathrm{MeV})$, respectively. As we see the predicted values are very similar for both sets of parameters and slightly smaller than the values in Refs. [5,6], $T_{P} \approx 100 \mathrm{MeV}$ and $\mu_{P} \approx 200-230 \mathrm{MeV}$. In this sense, we should remark that our model predicts a critical temperature at $\mu=0$ of about $100 \mathrm{MeV}$, somewhat below the values obtained in modern lattice simulations [17] which suggest $T_{c} \approx 140-190 \mathrm{MeV}$. In any case, our calculation seems to indicate that $\mu_{P}$ might be smaller than previously expected even in the absence of strangeness degrees of freedom.

It is interesting to discuss in detail the situation concerning the confining set. In this case we can find, for each temperature, the chemical potential $\mu_{d}$ at which confinement is lost. Following the proposal of Ref. [12], this corresponds to the point for which the self-energy at zero momentum reaches $\Sigma(0)_{\text {crit }}$ (cf. Eq. (16)). Using the values of $m_{c}$ and $\Lambda$ corresponding to Set I we get $\Sigma(0)_{\text {crit }}=267 \mathrm{MeV}$. For low temperatures, $\mu_{d}$ coincides with the chemical potential at which the chiral phase transition takes place. However, for a temperature close enough to that of the "end point", $\mu_{d}$ starts to be slightly smaller than the value of $\mu$ that corresponds to the chiral restoration. Above $T_{E}$ it is difficult to make an accurate comparison since, for finite quark masses, the chiral restoration proceeds through a smooth crossover. However, we can still study the situation in the chiral limit. In this case we find that, in the region where the chiral transition is of second order, deconfinement always occurs, for fixed $T$, at a lower value of $\mu$ than the chiral transition. The corresponding critical line is indicated by a dashed line in the left panel of Fig. 2. In any case, as we can see in this figure, the departure of the line of chiral restoration from that of deconfinement is in general not too large. This indicates that within the present model both transitions tend to happen at, approximately, the same point.

In conclusion, in this work we have investigated a nonlocal covariant extension of the NJL model at finite temperature and chemical potential. We have assumed that the corresponding four point vertex is separable in momentum space and that the regulator leads to an Euclidean quark propagator with an arbitrary (but discrete) number of poles in the complex plane. We have shown that the dependence on $\mu$ and $T$ can be expressed in terms of these poles and the corresponding residues. We have studied in detail the particular case of a Gaussian regulator, considering two different situations. In the first case, the Euclidean quark propagator does not have any purely 

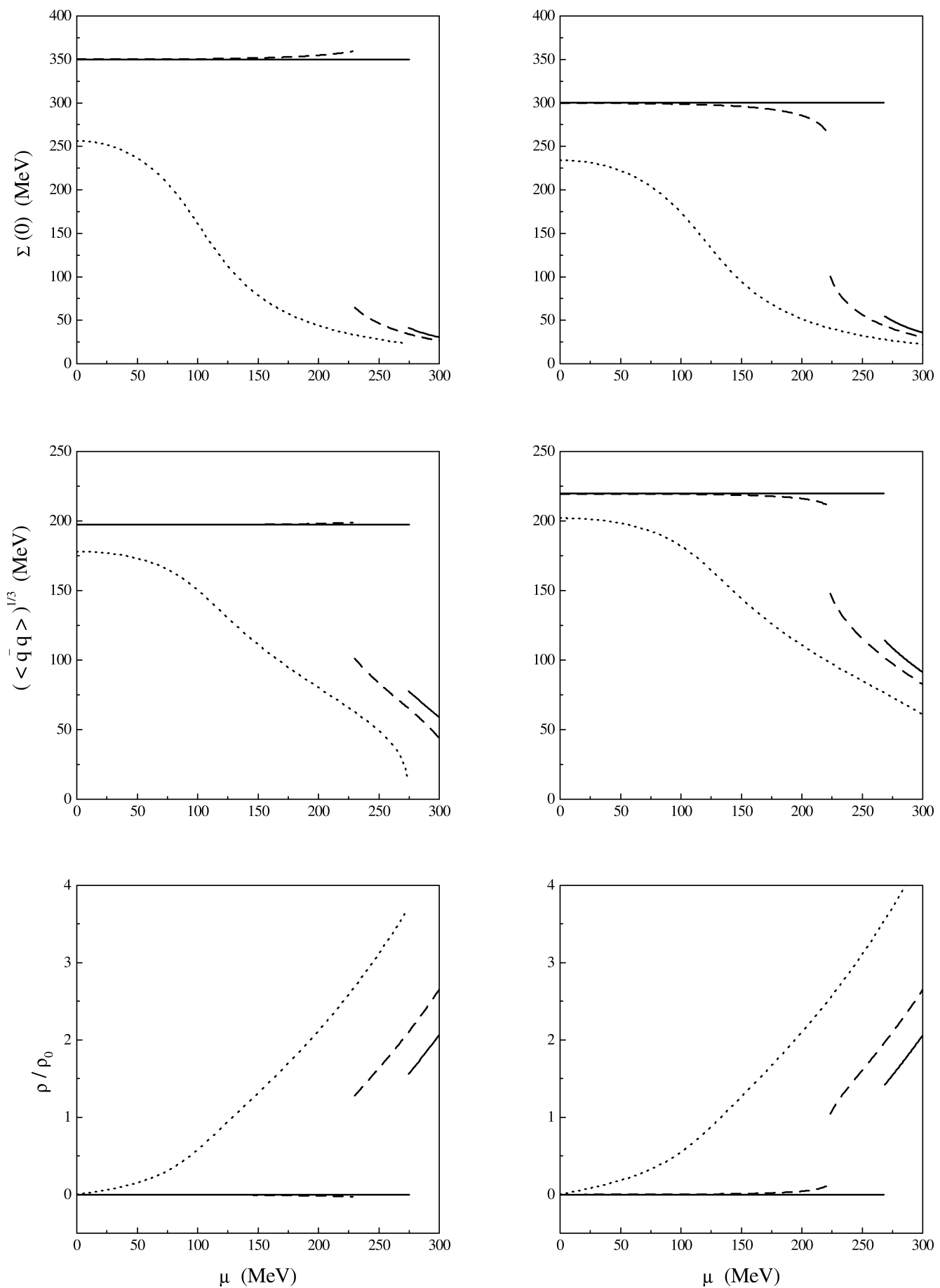

Fig. 1. Behaviour of the self-energy, the chiral condensate and the quark density as functions of the chemical potential for three representative values of the temperature. Full line corresponds to $T=0$, dashed line to $T=50 \mathrm{MeV}$ and dotted line to $T=100 \mathrm{MeV}$. The left panels display the results for Set I and the right panels those for Set II. The quark density $\rho$ is given with respect to nuclear matter density $\rho_{0}=1.3 \times 10^{6} \mathrm{MeV}^{3}$. 

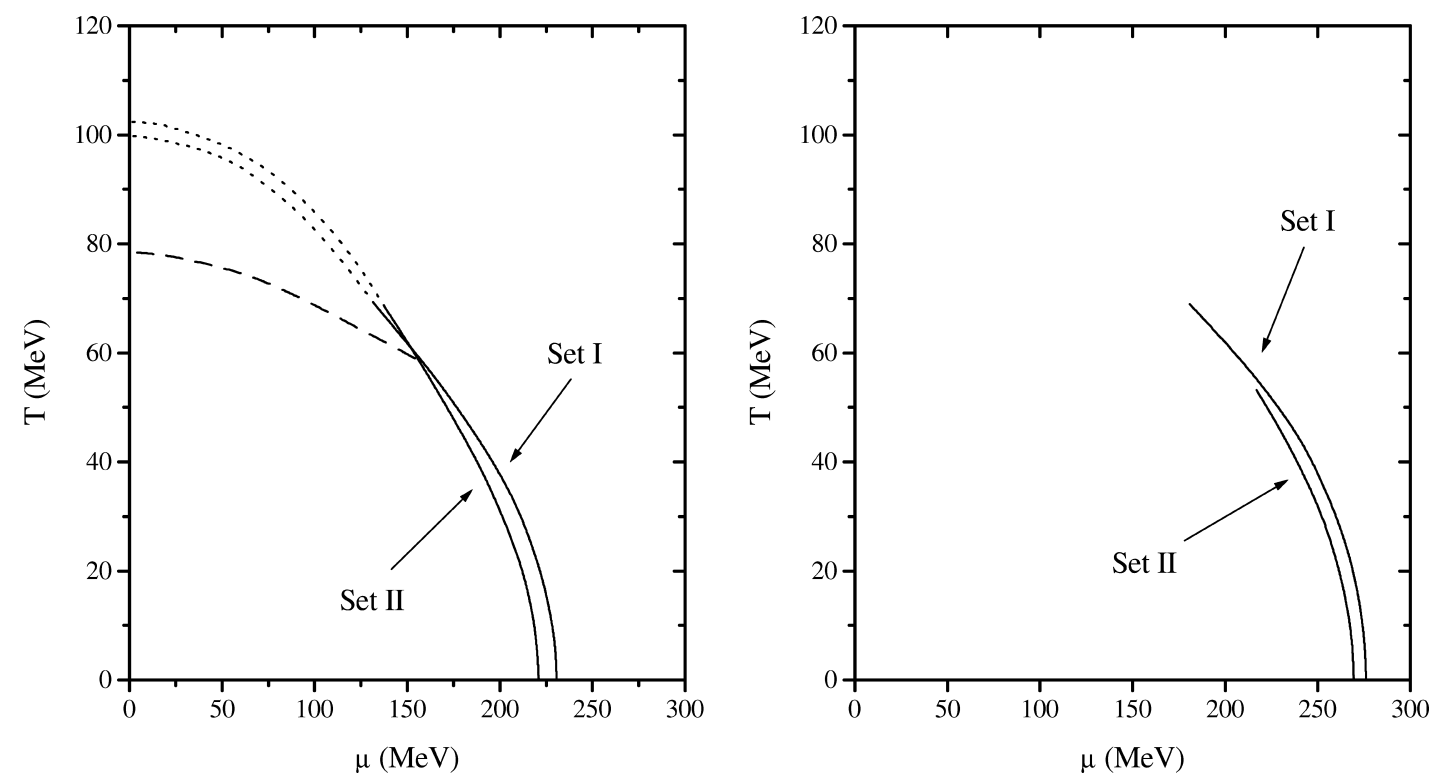

Fig. 2. Critical temperatures as a function of the chemical potential. The left panel corresponds to the chiral limit and the right panel to the case of finite quark masses. In the left panel, the dotted lines correspond to the region of the second order phase transition, while the full lines in both panels indicate the region where a first order transition occurs. The dashed line in the left panel indicates the critical line for deconfinement corresponding to Set I. For chemical potentials somewhat larger than $\mu_{P}$ this line coincides with that of the chiral restoration.

imaginary poles, which can be understood as a way of confinement. In the second case, this type of poles exists, thus quarks would be not confined. We have found that in both cases the behaviour of the physical quantities as a function of $T$ and $\mu$ is quite similar. In particular, for low values of $T$ the chiral transition is always of first order and, for finite quark masses, at certain "end point" the transition turns into a smooth crossover. In the chiral limit, this "end point" becomes a "tricritical" point. Our predictions for the position of these points are similar, although somewhat smaller, than previous estimates obtained in alternative models $[5,6]$. It is clear that several extensions of this work are possible. Firstly, it would be interesting to include interactions in the vector and axial-vector meson channels, in order to investigate meson properties close to the phase transition. Secondly, to study the possible existence of color superconductivity in this sort of models, interactions in the quark-quark channels should be incorporated. Finally, the extension to the $S U(3)$ sector would allow to understand the role of the strangeness in the characteristics of the chiral phase transition as described by nonlocal covariant models. We hope to report on these issues in forthcoming publications.

\section{Acknowledgements}

NNS wishes to thank O. Civitarese, H. Forkel and C.L. Schat for very enlightening discussions. The authors are also thankful to D. Blaschke for a very timely and useful correspondence. This work was supported in part by a grant from Fundación Antorchas, Argentina, and the grant PICT 03-00000-00133 from ANPCYT, Argentina.

\section{References}

[1] F. Wilczek, hep-ph/0003183; K. Rajagopal, hep-ph/0009058. 
[2] Y. Nambu, G. Jona-Lasinio, Phys. Rev. 122 (1961) 345;

Y. Nambu, G. Jona-Lasinio, Phys. Rev. 124 (1961) 246.

[3] U. Vogl, W. Weise, Prog. Part. Nucl. Phys. 27 (1991) 195;

S. Klevansky, Rev. Mod. Phys. 64 (1992) 649;

T. Hatsuda, T. Kunihiro, Phys. Rep. 247 (1994) 221.

[4] M. Aldorf, K. Rajagopal, F. Wilczek, Phys. Lett. B 422 (1998) 247;

M. Aldorf, K. Rajagopal, F. Wilczek, Nucl. Phys. B 537 (1999) 443;

R. Rapp, T. Schafer, E.V. Shuryak, M. Velkovsky, Phys. Rev. Lett. 81 (1998) 53.

[5] J. Berges, K. Rajagopal, Nucl. Phys. B 538 (1999) 215.

[6] M.A. Halasz, A.D. Jackson, R.E. Shrock, M.A. Stephanov, J.J.M. Verbaarschot, Phys. Rev. D 58 (1998) 096007.

[7] G. Ripka, Quarks Bound by Chiral Fields, Oxford University Press, Oxford, 1997.

[8] T. Schaefer, E. Schuryak, Rev. Mod. Phys. 70 (1998) 323.

[9] C.D. Roberts, A.G. Williams, Prog. Part. Nucl. Phys. 33 (1994) 477;

C.D. Roberts, S.M. Schmidt, Prog. Part. Nucl. Phys. 45S1 (2000) 1.

[10] E.R. Arriola, L.L. Salcedo, Phys. Lett. B 450 (1999) 225.

[11] G. Ripka, hep-ph/0003201;

R.S. Plant, M.C. Birse, hep-ph/0007340.

[12] R.D. Bowler, M.C. Birse, Nucl. Phys. A 582 (1995) 655;

R.S. Plant, M.C. Birse, Nucl. Phys. A 628 (1998) 607.

[13] D. Blaschke, G. Burau, Y.L. Kalinovsky, P. Maris, P.C. Tandy, nucl-th/0002024;

B. Szczerbińska, W. Broniowski, Acta Phys. Polon. B 31 (2000) 835.

[14] J.I. Kapusta, Finite-Temperature Field Theory, Cambridge Univ. Press, Cambridge, 1989.

[15] B. Golli, W. Broniowski, G. Ripka, Phys. Lett. B 437 (1998) 24.

[16] M. Stephanov, K. Rajagopal, E. Shuryak, Phys. Rev. Lett. 81 (1998) 4816; M. Stephanov, K. Rajagopal, E. Shuryak, Phys. Rev. D 60 (1999) 114028.

[17] F. Karsch, Nucl. Phys. (Proc. Suppl.) 83 (2000) 14; A. Ali Khan et al., Phys. Rev. D 63 (2001) 034502. 